\title{
Preoperative management using inhalation therapy \\ for pulmonary complications in lung cancer patients with chronic obstructive pulmonary disease
}

\author{
Kyoshiro Takegahara $^{1} \cdot$ Jitsuo Usuda $^{1} \mathbb{D} \cdot$ Tatsuya Inoue $^{1} \cdot$ Takayuki Ibi $^{1} \cdot$ Akira Sato $^{1}$
}

Received: 10 December 2016 / Accepted: 13 February 2017 / Published online: 9 March 2017

(C) The Author(s) 2017. This article is published with open access at Springerlink.com

\begin{abstract}
Objective This study aimed to investigate whether perioperative inhalations of long-acting beta-agonists (LABAs) or long-acting muscarinic antagonists (LAMAs) might decrease the incidence of postoperative complications in lung cancer patients with chronic obstructive pulmonary disease (COPD).

Methods We retrospectively analyzed 108 patients with COPD who underwent pulmonary resections for primary lung cancer at our hospital between January 2013 and January 2016 to determine the association between the incidence of postoperative complications (e.g., prolonged air leakage and pneumonia) and the use of LABAs or LAMAs. Results Thirty patients with COPD experienced postoperative complications (27.8\%): Fourteen patients had prolonged air leakages (more than 7 days), ten patients developed pneumonia. The frequency of these postoperative pulmonary complications was significantly higher among the patients with COPD (24/108 cases, 22.2\%), compared with the frequency among non-COPD patients (15/224 cases, 6.7\%). Inhaled bronchodilators, such as LAMA or LABA, were prescribed for 34 of the 108 patients with COPD; the remaining 74 patients were not treated with bronchodilators. Pulmonary complications were significant lower among the LAMA or LABA users (3/34 cases, 8.8\%) than among the untreated COPD patients (21/74 cases, $28.4 \%)$
\end{abstract}

Presented at The 68th Annual Scientific Meeting of The Japanese Association for Thoracic Surgery.

Jitsuo Usuda

jusuda@nms.ac.jp

1 Department of Thoracic Surgery, Nippon Medical School, 1-1-5 Sendagi, Bunkyo-ku, Tokyo 113-8603, Japan
Conclusion For lung cancer patients with COPD, preoperative management using LABA or LAMA bronchodilators and smoking cessation can reduce the frequency of postoperative pulmonary complications after surgical lung resection. LAMA or LABA inhalation might be useful for not only perioperative care, but also for the long-term survival of COPD patients after surgery.

Keywords Chronic obstructive pulmonary disease . Long-acting beta-agonist $\cdot$ Long-acting muscarinic antagonist $\cdot$ Lung cancer $\cdot$ Complications

\section{Introduction}

Chronic obstructive pulmonary disease (COPD) causes impaired pulmonary function as a symptom of occlusive disorder and is associated with a significantly high incidence of postoperative pulmonary complications, such as pneumonia, acute bronchitis, and atelectasis. Many patients with COPD have nonspecific airway hyperreactivity, suggesting the possible presence of bronchospasm or latent respiratory tract infection. To reduce the risk of postoperative complications, efforts should be made to alleviate peripheral airway obstruction and to reduce airway secretion [1, 2]. In Japan, with its aging society, the number of patients with lung cancer complicated with COPD is expected to further increase, while an increased incidence of multiple lung cancers makes the perioperative management of surgically treated patients with lung cancer very important [3]. Because the prognosis of patients with lung cancer complicated with COPD is reportedly poor, it is important to provide respiratory care not just during the perioperative period after pneumonectomy, but also for longer periods [4, 5]. The combination of smoking 
cessation, respiratory rehabilitation, and the use of longacting muscarinic antagonists (LAMAs) or long-acting beta-agonists (LABAs) has been reported to alleviate postoperative complications in patients with lung cancer complicated with COPD [4, 6-11]. The causal factors for airway obstruction in COPD are an irreversible decrease in elastic alveolar recoil, peripheral airway obstruction, and reversible contraction of the airway smooth muscle. The relaxation of airway smooth muscle is necessary for the resolution of airway obstruction. To treat the pathological symptoms of COPD, anticholinergics, which prevent acetylcholine from binding to muscarinic receptors, and beta- 2 agonists are used. Thus, acetylcholine and the muscarinic receptor system play important roles in the pathogenesis of COPD $[2,12,13]$.

Recently developed LABAs are superior to the previous ones in terms of their bronchodilation efficacy. The fourth edition of the Guidelines for the Diagnosis and Treatment of Chronic Obstructive Pulmonary Disease mentions that LAMAs and LABAs are comparable with respect to their usefulness for inhalation therapy and recommends inhalation therapy with LAMAs or LABAs $[13,14]$. In this study, we aimed to investigate whether the incidence of postoperative complications during the perioperative period could be reduced in patients with lung cancer complicated by COPD by not only providing smoking cessation services before surgery, but also by initiating the inhalation of LABAs or LAMAs.

\section{Patients and methods}

We defined ex-smokers as patients who had quit smoking at least 6 months before surgery; current smokers were defined as those who had quit smoking within 6 months before surgery. Patients with a smoking history and a forced expiratory volume in $1 \mathrm{~s} \mathrm{(FEV1.0)} \mathrm{to} \mathrm{forced}$ vital capacity (FVC) ratio of less than $70 \%$ were classified as COPD patients; the other patients were classified as non-COPD patients. Of the 332 patients who underwent surgical resections for primary lung cancer at our hospital between January 2013 and January 2016, the medical records of 108 patients who had COPD and a smoking history were retrospectively analyzed to determine the association between the incidence of postoperative complications (e.g., prolonged pulmonary fistulae and pneumonia) and the use of LABAs or LAMAs. We recommended inhalations to all the patients based on the diagnostic criteria of COPD and used them for the patients who agreed to the recommendation. The medical records of the remaining 224 non-COPD patients were also examined for comparison purposes. In addition, the use of LABAs or LAMAs started from at least 1 month
Table 1 Clinicopathological characteristics of the study population

\begin{tabular}{ll}
\hline Characteristics & \\
COPD(non-COPD) & $108(224)$ \\
Age (years) & $69.3(46-84)$ \\
Male/female & $86 / 22$ \\
Smoking (BI) & $1172.1(50-3480)$ \\
Current/ex-smoker & $45 / 63$ \\
FEV1.0/FVC & $61.4(26.8-69.9)$ \\
c-stage(IA/IB/IIA/IIB/IIIA) & $49 / 24 / 16 / 7 / 12$ \\
Operation methods & \\
Wedge resection & 11 \\
Segmentectomy & 3 \\
Lobectomy & 92 \\
Pneumonectomy & 2 \\
Histological type & \\
Adeno & 53 \\
Squamous & 38 \\
Large & 4 \\
Small & 1 \\
LCNEC & 3 \\
Others & 9 \\
\end{tabular}

ago of the operation and continued after operation based on the fourth edition of the Guidelines for the Diagnosis and Treatment of Chronic Obstructive Pulmonary Disease.

\section{Results and discussion}

Among the 108 COPD patients, there were 86 men and 22 women, with a mean age of 69.3 years (range 46-84 years). The mean Brinkman index was 1172.1 (range 50-3480). There were 45 current smokers and 63 ex-smokers. The mean FEV1.0/FVC was $61.4 \%$ (range 26.8-69.9\%) (Table 1). The performed surgical procedures were a partial resection in 11 patients, a pulmonary segmentectomy in 3 patients, a lobectomy in 92 patients, and a pneumonectomy in two patients. The histological types were adenocarcinoma in 53 patients, squamous

Table 2 Postoperative complications

\begin{tabular}{llll}
\hline & COPD(108) & Non-COPD(224) & $P$ value \\
\hline Prolonged air leakage & $14(13.0 \%)$ & $9(4.0 \%)$ & 0.0055 \\
Pneumonia & $10(9.3 \%)$ & $6(2.7 \%)$ & 0.0188 \\
IP & 0 & 2 & 0.8196 \\
Arrhythmia & 2 & 4 & 1 \\
Chylothorax & 2 & 2 & 0.831 \\
Wound infection & 2 & 3 & 1
\end{tabular}


Table 3 (A) Postoperative complications of COPD patients. (B) Postoperative complications of current smoker

\begin{tabular}{llll}
\hline A & LABA/LAMA & No medications & $P$ value \\
\hline Prolonged air leakage + Pneumonia & $3 / 34(8.8 \%)$ & $21 / 74(28.4 \%)$ & 0.0433 \\
\hline Prolonged air leakage + Pneumonia & Current smoker & Ex-smoker & $P$ value \\
\hline B & $9 / 45(20.0 \%)$ & $15 / 63(23.8 \%)$ & 0.8144 \\
\hline Prolonged air leakage + Pneumonia & LABA/LAMA & No medications & $P$ value \\
\hline
\end{tabular}

cell carcinoma in 38 patients, adenosquamous carcinoma in 5 patients, large cell neuroendocrine carcinoma in 3 patients, large cell carcinoma in 4 patients, small cell carcinoma in one patient, and pleomorphic carcinoma in 4 patients (Table 1). There were no surgical or in-hospital deaths. Postoperative complications were observed in 30 patients. The patients received wedge resection are not included in these 30 patients. Regarding the incidence of pulmonary complications, such as prolonged air leakage and pneumonia, 24 patients $(22.2 \%)$ with COPD and 15 (6.7\%) non-COPD patients experienced complications. The incidence was significantly higher among the COPD patients than among the non-COPD patients. No significance differences in the incidences of arrhythmia, wound infection, or other complications were observed between the COPD and non-COPD patients (Table 2). The incidence of prolonged air leakage and pneumonia was significantly lower among the 34 patients who received LABAs or LAMAs ( 3 patients, $8.8 \%$ ) than among the 74 patients who did not receive these drugs (21 patients, $28.4 \%$ ) (Table 3 ).

The COPD patients included 45 current smokers; 24 of these patients had undergone smoking cessation therapy for at least 1 month before surgery, respiratory rehabilitation, and the inhalation of LABAs or LAMAs. Three of the twenty-four patients $(12.5 \%)$ who underwent inhalation therapy as part of their preoperative management developed prolonged air leakages or pneumonia, whereas six of the twenty-one current smokers (28.6\%) who did not 66 receive inhalation therapy developed these pulmonary complications. The incidence of pulmonary complications was lower among current smokers who had started inhalation therapy as part of their preoperative management; however, there was no significant difference statistically (Table 3).

In the present study, examining lung cancer patients with COPD, preoperative management that included the inhalation of LABAs or LAMAs reduced the frequency of postoperative pulmonary complications after surgical resection. For the reason why complications decrease by preoperative inhalation therapy, we think that perioperative sealing tests go well and that drainage of the sputum from peripheral airway goes well by inhalation therapy. Other possibility was that the inhalation of LABA or LAMA prevented the pneumonia at the peripheral lung by anti-inflammatory effect. We think that further detailed examination will be necessary in future. Whereas, it was reported that inhalation therapy is not necessarily effective as Yamanashi et al. reported. This report shows that there was no significant difference about inhalation therapy using corticosteroids (ICSs) [15]. As for the recent LABA, effects come out early and effects are high [9, 13, 14]. However, we think that the accumulation of cases is necessary for comparison.

LABA and LAMA inhalants can be adapted for the management of perioperative care. For elderly patients with lung cancer, especially those with concurrent COPD, not only perioperative but also long-term respiratory care, including the administration of LABAs or LAMAs, might be necessary. Further prospective studies may be needed for the long-term survival of lung cancer patients with COPD after surgical resections.

Acknowledgements This work was supported in part by a Grant-inAid for Scientific Research (C) from the Japan Society for the Promotion of Science (JSPS), KAKENHI 16K10693 (J.U.), and supported by the Research on Development of New Medical Devices from the Japan Agency for Medical Research and development (AMED), 16hk0102025h0002 (J.U.).

\section{Compliance with ethical standards}

Conflict of interest All the authors have declared no competing interest.

Open Access This article is distributed under the terms of the Creative Commons Attribution 4.0 International License (http:// creativecommons.org/licenses/by/4.0/), which permits unrestricted use, distribution, and reproduction in any medium, provided you give appropriate credit to the original author(s) and the source, provide a link to the Creative Commons license, and indicate if changes were made. 


\section{References}

1. Zhai R, Yu X, Shafer A, et al. The impact of coexisting copd on survival of patients with early-stage non-small cell lung cancer undergoing surgical resection. Chest. 2014;145:346-53.

2. Qaseem A, Wilt TJ, Weinberger SE, et al. Diagnosis and management of stable chronic obstructive pulmonary disease: a clinical practice guideline update from the American College of Physicians, American College of Chest Physicians, American Thoracic Society, and European Respiratory Society. Ann Intern Med. 2011;155:179-91.

3. Committee for Scientific Affairs, The Japanese Association for Thoracic Surgery, Masuda M, Kuwano H, Okuhara M, et al. Thoracic and cardiovascular surgery in Japan during 2012. Gen Thorac Cardiovasc Surg. 2014;62:734-64.

4. Leo F, Venissac N, Pop D, et al. Postoperative exacerbation of chronic obstructive pulmonary disease. Does it exist? Eur J Cardiothorac Surg. 2008;33:424-29.

5. Kiri VA, Fabbri LM, Davis KJ, et al. Inhaled corticosteroids and risk of lung cancer among COPD patients who quit smoking. Respir Med. 2009;103:85-90.

6. Yoshida Y, Kage H, Murakawa T, et al. Worse prognosis for stage IA lung cancer patients with smoking history and more severe chronic obstructive pulmonary disease. Ann Thorac Cardiovasc Surg. 2015;21:194-200.

7. Calverley PM, Anderson JA, Celli B, et al. Salmeterol and fluticasone propionate and survival in chronic obstructive pulmonary disease. N Engl J Med. 2007;356:775-89.

8. Bolukbas S, Eberlein M, Eckhoff J, et al. Short-term effects of inhalative tiotropium/formoterol/budenoside versus tiotropium/formoterol in patients with newly diagnosed chronic obstructive pulmonary disease requiring surgery for lung cancer: a prospective randomized trial. Eur J Cardiothorac Surg. 2011;39:995-1000.

9. Divisi D, Di Francesco C, Di Leonardo G, et al. Preoperative pulmonary rehabilitation in patients with lung cancer and chronic obstructive pulmonary disease. Eur J Cardiothorac Surg. 2013;43:293-96.

10. Stefanelli F, Meoli I, Cobuccio R, et al. High-intensity training and cardiopulmonary exercise testing in patients with chronic obstructive pulmonary disease and non-small-cell lung cancer undergoing lobectomy. Eur J Cardiothorac Surg. 2013;44:260-65.

11. Shiono S, Katahira M, Abiko M, et al. Smoking is a perioperative risk factor and prognostic factor for lung cancer surgery. Gen. Thorac Cardiovasc Surg. 2015;63:93-8.

12. Struckmann N, Schwering S, Wiegand S, et al. Role of muscarinic reseptor subtypes in the constriction of peripheral airways: studies on receptor-dificient mice. Mol Pharmacol. 2003;64:1444-51.

13. Kurosawa $\mathrm{H}$, et al. Outline of JRS COPD guideline Ed. 4. Nihon Rinsho. 2016;74:728-32.

14. Decramer ML, Chapman KR, Dahl R, et al. Once-daily indacterol versus tiotropium for patients with chronic obstructive pulmonary disease (INVIGORATE): a randomaised, blinded, parallel-group study. Lancet. Respir Med. 2013;1:524-33.

15. Yamanashi K, Marumo S, Shoji $\mathrm{T}$, et al. The relationship between perioperative administration of inhaled corticosteroid and postoperative respiratory complications after pulmonary resection for non-small- cell lung cancer in patients with chronic obstructive pulmonary disease. Gen Thorac Cardiovasc Surg. 2015;63(12):652-9. 